\title{
Asthma diagnosis and treatment - 1016. Is atopy in people aged 40 and over related to fixed airflow obstruction?
}

\author{
Michael J Abramson ${ }^{1 *}$, Brett G Toelle ${ }^{2}$, Alan L James ${ }^{3}$, Richard Wood-Baker ${ }^{4}$, Deborah Burton ${ }^{5}$, Wei Xuan², \\ David P Johns ${ }^{6}$, A Sonia Buist ${ }^{7}$, Guy B Marks ${ }^{2}$ \\ From 2nd WAO International Scientific Conference (WISC 2012) \\ Hyderabad, India. 6-9 December 2012
}

\section{Background}

The Tucson and Normative Ageing studies showed atopy was not related to airflow obstruction in later life, when known asthmatics were excluded. However these studies were based on pre-bronchodilator spirometry.

\section{Aim}

To examine independent associations between atopy and post-bronchodilator airflow obstruction in people aged 40 years and over.

\section{Methods}

We used data from the BOLD-Australia study. Persons aged 40 years and over were randomly selected from the electoral roll, either directly or via a two stage sampling procedure, in centres based in four Australian States. Spirometry was performed before and after administration of salbutamol $200 \mu \mathrm{g}$, according to a standardised protocol with careful attention to quality control. Subjects with post-bronchodilator $\mathrm{FEV}_{1} / \mathrm{FVC}$ ratio $<0.7$ were classified as GOLD stage 1 or higher. Subjects who also had $\mathrm{FEV}_{1}<80 \%$ predicted were classified as GOLD stage 2 or higher. Subjects who had a post-bronchodilator increase in $\mathrm{FEV}_{1}>12 \%$ of pre-bronchodilator value and $>200 \mathrm{ml}$ were classified as "reversible". Atopy was assessed by skin prick tests (SPTs) to house dust mites (D. pteronyssinus and D. farinae), cat, dog, cockroach, Alternaria, Aspergillus, rye grass and mixed grass pollens. Subjects with any allergen SPT $\geq 4 \mathrm{~mm}$ were classified as atopic.

${ }^{1}$ Epidemiology \& Preventive Medicine, Monash University, Australia Full list of author information is available at the end of the article

\section{Results}

Post-bronchodilator spirometry and SPT data were available for 2,767 subjects ( $51.4 \%$ female, $12.1 \%$ aged $\geq 75$ ). The prevalence of GOLD stage 1 or higher was $15.9 \%$, the prevalence of GOLD stage 2 or higher was $6.8 \%$, and $4.7 \%$ had bronchodilator reversibility. One in nine subjects selfreported current asthma and 50.5\% had ever been smokers. The prevalence of atopy in this population was $42.7 \%$.

The associations between atopy and airflow obstruction did not differ by self-reported asthma or smoking status (all interactions $\mathrm{p}>0.2$ ). However atopy was associated with GOLD stage 2 after adjustment for sex, self-reported current asthma and smoking status $(\mathrm{OR}=1.56,95 \% \mathrm{CI}$ 1.13-2.17). As expected, atopy was related to the presence of bronchodilator reversibility (OR=1.89, 95\%CI 1.32-2.72).

\section{Conclusions}

The apparent association of atopy with fixed airflow obstruction using post-bronchodilator spirometry in people aged 40 years and over may be due to clinical heterogeneity.

\section{Author details}

'Epidemiology \& Preventive Medicine, Monash University, Australia. ${ }^{2}$ Woolcock Institute of Medical Research, Sydney, Australia. ${ }^{3}$ Sir Charles Gairdner Hospital, Perth, Australia. ${ }^{4}$ Royal Hobart Hospital, Hobart, Australia. ${ }^{5}$ Charles Sturt University, Orange, Australia. ${ }^{6}$ Menzies Research Institute, Hobart, Australia. ${ }^{7}$ Oregon Health \& Science University, Portland, OR, USA.

Published: 23 April 2013

\section{doi:10.1186/1939-4551-6-S1-P16}

Cite this article as: Abramson et al: Asthma diagnosis and treatment -

1016. Is atopy in people aged 40 and over related to fixed airflow obstruction? World Allergy Organization Journal 2013 6(Suppl 1):P16.

\section{C)

(c) 2013 Abramson et al; licensee BioMed Central Ltd. This is an Open Access article distributed under the terms of the Creative Commons Attribution License (http://creativecommons.org/licenses/by/2.0), which permits unrestricted use, distribution, and reproduction in any medium, provided the original work is properly cited. 